\title{
Hard Decision Fusion based Cooperative Spectrum Sensing in Cognitive Radio System
}

\author{
N. Armi, N.M. Saad \& M. Arshad \\ Department of Electrical and Electronics Engineering \\ PETRONAS University of Technology \\ Bandar Seri Iskandar, 31750, Tronoh, Perak Darul Ridzuan, Malaysia
}

\begin{abstract}
Cooperative spectrum sensing was proposed to combat fading, noise uncertainty, shadowing, and even hidden node problem due to primary users (PUs) activity that is not spatially localized. It improves the probability of detection by collaborating to detect PUs signal in cognitive radio (CR) system as well. This paper studies cooperative spectrum sensing and signal detection in CR system by implementing hard decision combining in data fusion centre. Through computer simulation, we evaluate the performances of cooperative spectrum sensing and signal detection by employing OR and AND rules as decision combining. Energy detector is used to observe the presence of primary user (PU) signal. Those results are compared to non-cooperative signal detection for evaluation. They show that cooperative technique has better performance than non-cooperative. Moreover, signal to noise ratio (SNR) with greater than or equal $10 \mathrm{~dB}$ and 15 collaborated users in $\mathrm{CR}$ system has optimal value for probability of detection.
\end{abstract}

Keywords: cognitive radio (CR); cooperative; hard decision; OR and AND rule; spectrum sensing.

\section{$1 \quad$ Introduction}

Today's wireless networks are characterized by fixed spectrum assignment policy. As increasing demand for frequency spectrum and limited resource availability, FCC decided to make a paradigm shift by allowing more number of unlicensed users to transmit their signals in licensed bands to efficiently utilize the available spectrum.

Cognitive radio has been proposed as a means to overcome spectrum scarcity in wireless communication. It has two important functionalities: spectrum sensing and adaptation. A secondary terminal first senses the spectrum environment in order to learn the frequency spectrum unoccupied by PUs. Once such a spectrum hole is found, the secondary user adapts its transmission power, frequency band, modulation, etc., so that it minimizes the interference to the primary users. Even after starting the transmission, the secondary user should be able to detect or predict the appearance of a primary user so that it makes the 
spectrum available for the primary user. Basically, the primary users should not change their communication infrastructure due to these operations. Thus, these sensing (including the detection) and adaptation of the secondary users must be performed independently of the primary users. Moreover, cognitive radio senses the spectrum environment over a wide range of frequency band and exploits this information to opportunistically provide wireless links that not only can meet the best demand of the user, but also of its radio environments.

Cooperative spectrum sensing was proposed to overcome noise uncertainties, fading and shadowing in primary user signal detection. It can be as a solution to hidden node problem and decrease sensing time as well [1]. In this technique, CR users/nodes are collaborated to sense spectrum hole and detect PUs signal. Then, with or without sharing local detection information among users, they forward them to data fusion centre. The fusion centre decides the final result in accordance with the decision rules whether primary signal is present or absent.

The cooperative spectrum sensing based on SNR comparison was studied by Yi Zheng, et al. [2]. They proposed scheme by selecting CR user with better SNR to forward their detection results to fusion centre. By this way can greatly increase probability of detection and reduce node number for final decision in data fusion centre. However, they presented the results by using probability of detection and probability of false alarm as a metric.

In this paper, we model CR user populated in the area of PU transmitter to detect primary user signal. The distances between $\mathrm{CR}$ users to primary transmitter and data fusion center are not considered. Each CR user detects PU signal and forwards their information of local detection to the data fusion centre. We use two decision rules, OR and AND rules for final decision [3]. Probability of detection and SNR are used as metrics to evaluate the performance of cooperative spectrum sensing.

The rest of this paper is organized as follows. In section 2 decision fusion is introduced. System model is presented in section 3. It briefly describes noncooperative and cooperative signal detection. Section 4 is the evaluation performance of non-cooperative and cooperative model, OR and AND rule respectively. Finally, we draw the conclusion in section 5.

\section{Decision Fusion}

In order to realize the cooperative detection among CR users, the spectrum sensing and signal detection information over individual users should be sent to a fusion centre for further process and the fusion centre makes the final decision whether primary user signal is present or absent. Since we discuss cooperative 
spectrum sensing under communication bandwidth constraints, it is proper that all cognitive radio users send their one-bit decision on spectrum sensing to fusion centre based on their local observations.

As described in Figure 1, information of local signal observation from all cognitive users transmits to data fusion centre. They forward 1-bit local detection to avoid communication overhead when $\mathrm{CR}$ users increased. Then, the final decision is performed whether signal is present $\left(\mathrm{H}_{1}\right)$ or absent $\left(\mathrm{H}_{0}\right)$ by regarding to decision rule

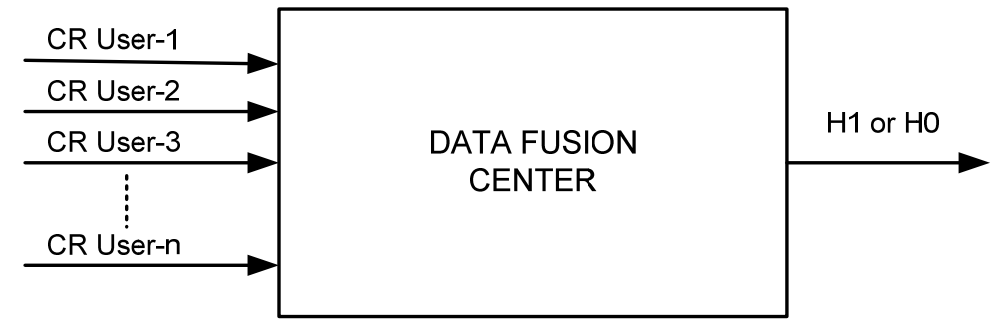

Figure 1 Data fusion centre.

There are two decision fusions commonly used in cooperative spectrum sensing, hard and soft decision. Hard decision is the one in which the individual cognitive radio makes the one-bit decision regarding the existence of the primary user. The bit-1 indicates that primary user uses spectrum channel, so that cognitive radio user cannot access. Spectrum channel is available to be accessed if cognitive radio user makes bit 0 . After observing the primary user signal, the local detection forwards them to data fusion centre for further process. The final decision then is taken by combining all local detection/observation. The two simple rules of hard decision are OR and AND rule. Under OR rule, at least one of the CR users involved in sensing decides that primary user is present. Whereas AND rule decides primary user is present when primary signal is detected by all cognitive radio users or in other word that all local decision of cognitive radio user is $\mathrm{H}_{1}$. In the case of soft decision, the decision is taken by correlating the measurement made by individual users in signal detection. It is more accurate than hard decision. However, it will cause data transmission overhead when number of CR users increase. In this case of study, we focus on hard decision combining where individual users forward their one bit decision to fusion centre.

The results presented in [4-5] show that soft decision combining outperforms hard decision combining in terms of the probability of miss-detection. On the other hand, hard decisions combining obtain as good results as soft decisions when the number of collaborated users is increased [6]. 


\section{$3 \quad$ System Model}

\subsubsection{Non-cooperative Spectrum Sensing}

In non-cooperative spectrum sensing, PUs signal are detected independently by $\mathrm{CR}$ user. Each user determines the presence and absence of PUs individually and acts accordingly.

As shown in Figure 2, CR users detect primary signal and decide whether signal is present or not by themselves. However, this technique cannot detect primary signal properly due to fading and shadowing. As shown in the figure, CR user-2 can detect primary transmitter signal more accurately than the other users because CR user 2 detects signal in line of sight (LOS) condition.

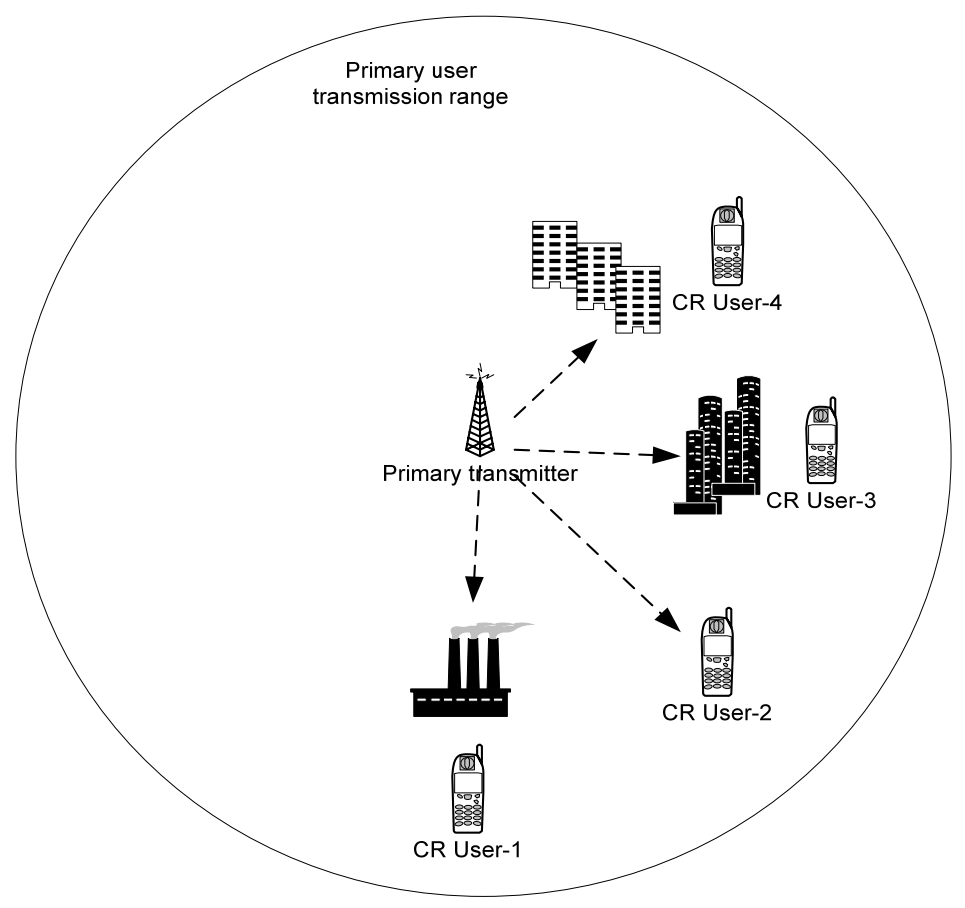

Figure 2 Model of non-cooperative technique.

There are number of techniques proposed for identifying the presence of PU signal transmissions such as matched filter, cyclostationary and energy detector. Energy detector requires no prior knowledge of the signal and is less complex than the other detectors. However, it has a limit on the required amount of 
signal SNR (SNR wall) [6-7]. A matched filter requires prior knowledge of the signal that it is used to detect the primary user signal. It is obtained by correlating the sensed signal with an already known signal. Furthermore, the cyclostationarity based detection algorithms can differentiate noise from primary users' signals. This is a result of the fact that noise is wide-sense stationary (WSS) with no correlation while modulated signals are cyclostationary with spectral correlation due to the redundancy of signal periodicities. However, it also requires prior knowledge of signal.

In this study, we use energy detector for signal detection due to its low complexity. The simplified block diagram of signal detector is shown in Figure 3.

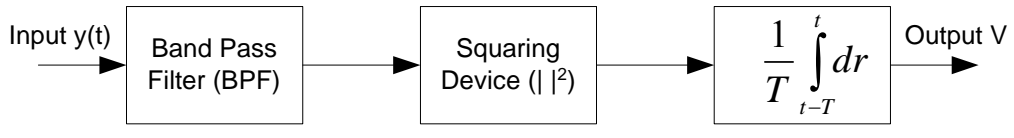

Figure 3 Block diagram of energy detector.

As described in the figure, the input signal $y(t)$ is filtered with a Band Pass Filter (BPF) in order to limit the noise and to select the bandwidth of interest. The noise in the output of the filter has a band-limited, flat spectral density.

The next blocks are a squaring device and a finite time integrator. The output of squaring device is further processed by integrator to obtain $V$ as formulated bellows [8]:

$$
V=\frac{1}{T} \int_{t-T}^{t}|y(r)|^{2} d r
$$

Finally, this output signal $V$ is compared to the threshold in order to decide whether a signal is present or not. The threshold is set according to statistical properties of the output $V$ when only noise is present.

\subsubsection{Cooperative Spectrum Sensing}

Cooperation is proposed as a solution to problems that arise in spectrum sensing due to noise uncertainty, fading, and shadowing. Cooperative sensing decreases the probabilities of miss-detection and false alarm considerably. In addition, cooperation can solve hidden primary user problem and it can decrease sensing time [9-11]. 
In this technique, the cognitive radio users are populated in the range of primary transmitter to perform its individual signal detection using some detection methods and determine the reliability of its own detection results. We adopt hard decision fusion method for this evaluation in which users send a binary local decision to data fusion centre.

Figure 4 shows the system model of cooperative signal detection where only one cognitive radio user could be able to detect the primary signal. The other cognitive radio users are not able to distinguish existence of the primary signal by fading and shadowing effect. The users are populated in the range of primary transmitter. Under this condition, it is expected that it can improve the signal detection probability. Collaboration among cognitive radio users is theoretically more accurate and convenient.

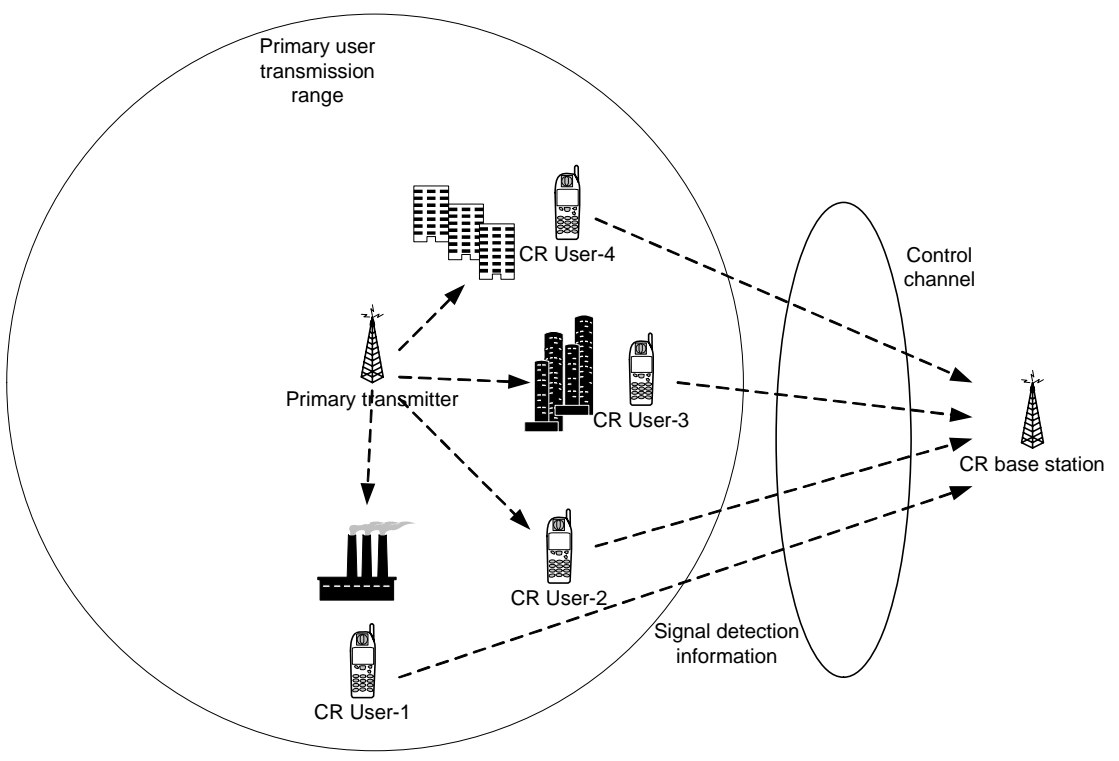

Figure 4 Cooperative signal detection model.

In order to improve probability of signal detection, the data fusion centre collects local signal detection information of each cognitive radio users and performs final decision in accordance with the decision rules.

Hard decision fusion shares their final binary bit to minimize communication overhead. The data fusion centre receives local decision from number of users and decides that signal is present $\left(\mathrm{H}_{1}\right)$ when total sum of user number decision 
is $\mathrm{H}_{1}$. It means that at least one $\mathrm{CR}$ user detects the primary transmitter signal and forwards 1-bit local detection. This fusion rule is known as OR rule. The AND rule decides primary signal is present when all local detection of CR users are $\mathrm{H}_{1}$.

In signal detection using hard decision based cooperative spectrum sensing, detection probability $\left(C_{d}\right)$ and false alarm probability $\left(C_{f a}\right)$ for OR rule are formulated as follows [12]:

$$
\begin{aligned}
& C_{d}=1-\prod_{1}^{n}\left(1-P_{d, k}\right) \\
& C_{f a}=1-\prod_{1}^{n}\left(1-P_{f a, k}\right)
\end{aligned}
$$

Furthermore, detection probability and false alarm probability by employing AND rule are given as follows [12]:

$$
\begin{aligned}
& C_{d}=\prod_{1}^{n} P_{d, k} \\
& C_{f a}=\prod_{1}^{n} P_{f a, k}
\end{aligned}
$$

where $P_{d, k}$ and $P_{f a, k}$ are detection probability and false alarm probability of the kth cognitive user, respectively. They can be computed using central chi-square (or gamma) PDF with $N$ degrees of freedom [13].

$$
P_{f}=P\left\{Y>\lambda \mid H_{o}\right\}=\frac{\Gamma\left(N / 2, \frac{\lambda}{2 \sigma^{2}}\right)}{\Gamma(N / 2)}
$$

where $\Gamma(. ., .$.$) and \Gamma()$ are the incomplete and complete gamma function respectively. In energy detector, this function is represented by the following operation [14]: 1) sampling the received signal and passing through an FFT device to obtain the signal spectrum, 2) the peak of the spectrum is then located and windowed, and finally, 3) the signal energy is then collected in the frequency domain and binary decision is created by comparing this energy to threshold value. Then, $N$ is degrees of freedom, $\sigma^{2}$ is noise variance of communication, $Y$ is a decision statistic, $\lambda$ is the decision threshold, and $H_{0}$ stand for the hypothesis: no signal transmitted. On the other hand, probability of detection can be computed using non-central chi-square PDF with $N$ degrees of freedom [13]. 


$$
P_{d}=P\left\{Y>\lambda \mid H_{1}\right\}=Q_{N / 2}\left(\sqrt{\frac{S^{2}}{\sigma^{2}}}, \sqrt{\frac{\lambda}{\sigma^{2}}}\right)
$$

where $\operatorname{Qm}(. .$.$) is Marcum Q-function, and H_{1}$ stand for the hypotheses: signal transmitted. The parameter $S^{2}=\sum_{i=0}^{N / 2} A_{i}$ is called the non-centrality parameter of the distribution and $\mathrm{A}$ is signal amplitude. These equations are valid for simply energy detector.

\section{$4 \quad$ Numerical Results and Evaluation}

As mentioned earlier, cooperative technique was proposed to combat noise uncertainty, fading, and shadowing. These all can cause sensing errors such as false detection and miss-identification. False detection senses idle states as a busy channel and CR users refrain to transmit data. On the other hand, missidentification senses busy states as an idle channel and cause CR users collide to PU transmission. Figure 5 shows cooperative technique compared with non cooperative one. We have different value of detection probability 0.2 at $\mathrm{SNR}=0$ $\mathrm{dB}$ and the values increased as SNR improved. However, when SNR greater than and equal $10 \mathrm{~dB}$, detection probability relatively close and equal for both of cooperative and non cooperative. It means that cooperative technique can be effectively and efficiently implemented when SNR lower than $10 \mathrm{~dB}$. In these values, cooperative technique has better values significantly than non cooperative one.

There are two rules commonly used in hard decision combining based cooperative spectrum sensing. OR rule decides $\mathrm{H} 1$ when at least one user detects primary user signal while AND rule decides $\mathrm{H} 1$ if all cognitive radio users forward their bit-1 local detections. Through computer simulation, we model cooperative spectrum sensing and obtain numerical results.

First, the OR and AND rule are studied. We define SNR values from 0-10 dB. We use this metric to indicate that $\mathrm{CR}$ users experience different channel fading. The information of local detection from each cognitive radio users are forwarded to data fusion centre and combined to obtain final decision. The simulation is performed by using probability of detection as a metric at different SNR values. 


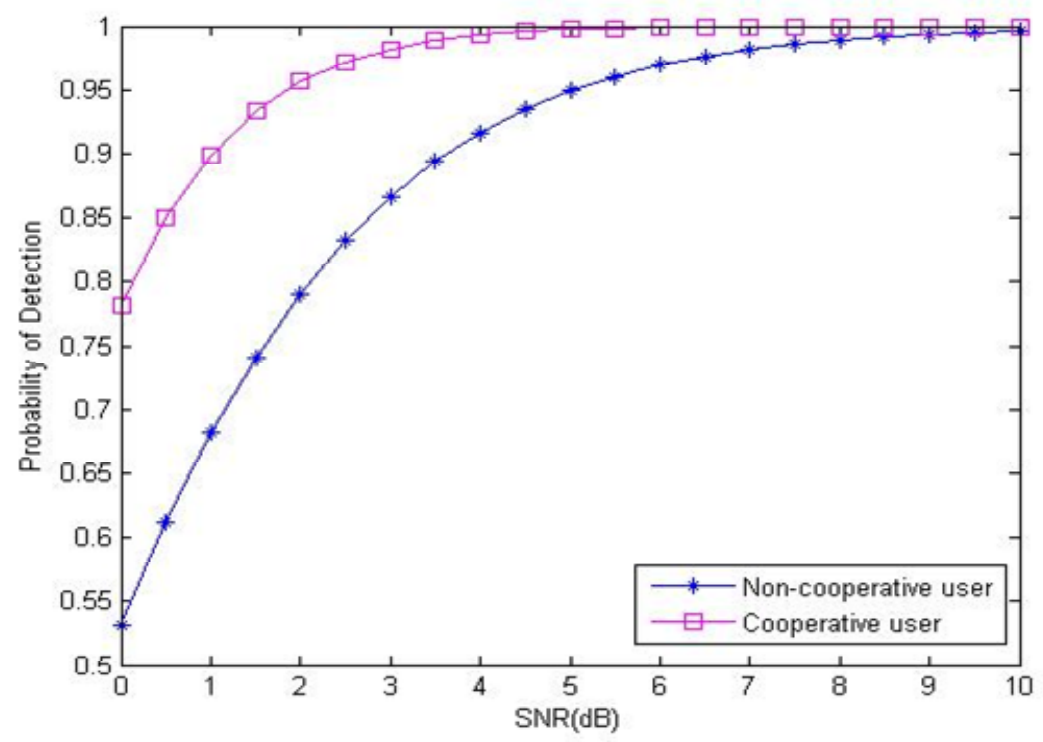

Figure 5 The performance comparison between cooperative and non cooperative.

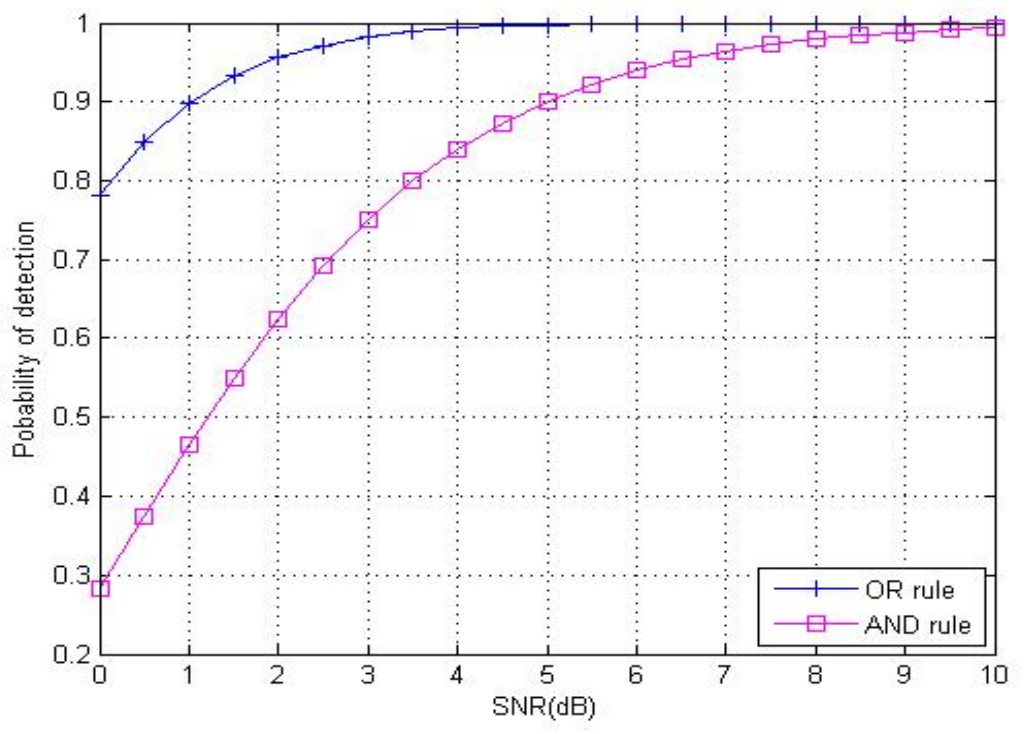

Figure 6 Probability of detection for OR and AND rule. 
Figure 6 describes the probability of detection by employing OR and AND rule. We assume 2 CR users are collaborated to detect primary user signal. As shown in the figure that OR rule has better probability of detection than AND rule. The data fusion centre decides $\mathrm{H}_{1}$ when at least there is one CR user detects primary user signal for OR rule while in AND rule, all local detection of CR users must be $\mathrm{H}_{1}$ to decide the presence of primary user signal. However, the figure shows when SNR value is greater than $10 \mathrm{~dB}$, both of the rules has an optimal probability of detection.

Then, we evaluate probability of detection with different number of cognitive radio users in each decision fusion rule. Number of 2 and 4 collaborated users is implemented for this evaluation. Figure 7 describes the probability of detection that employs OR rule with 2 and 4 collaborated users. Likewise, noncooperative signal detection technique is simulated as comparison.

As shown in the figure that number of 2 and 4 collaborated users improves the probability of detection. In case of low SNR, number of 4 collaborated users gives better value than the others. The low SNR is caused by propagation loss such as fading and shadowing. However, when SNR value is greater than 10 $\mathrm{dB}$, probability of detection obtain an optimal value relatively for both of noncooperative and cooperative cases.

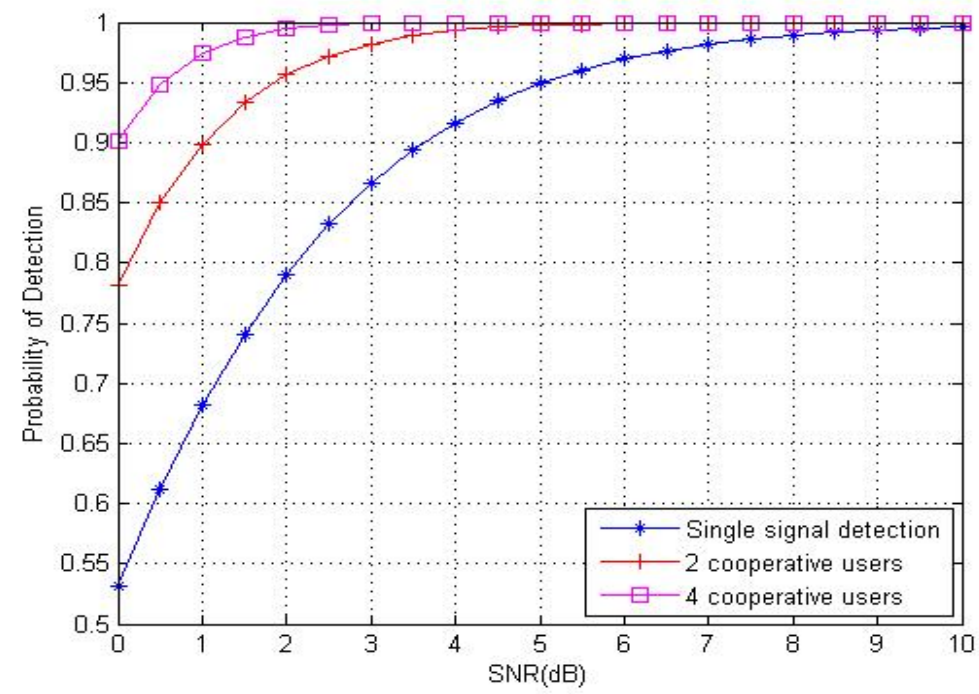

Figure 7 Probability of detection for OR rule. 
The evaluation of detection probability by employing AND rule is shown in Figure 8 . The 2 and 4 number of collaborated users is represented for evaluation and compares to non-cooperative signal detection. As described in the figure, when employing AND rule, non-cooperative case has better probability of detection values than the others. Increasing number of collaborated user causes probability of detection values become low in comparison with non-cooperative case. In this rule, data fusion centre decides the presence of primary user signal when all local detection of cognitive radio users forward bit- $1\left(\mathrm{H}_{1}\right)$. The same as previous results, when SNR value is greater than $10 \mathrm{~dB}$, probability of detection achieves an optimal and equal value relatively.

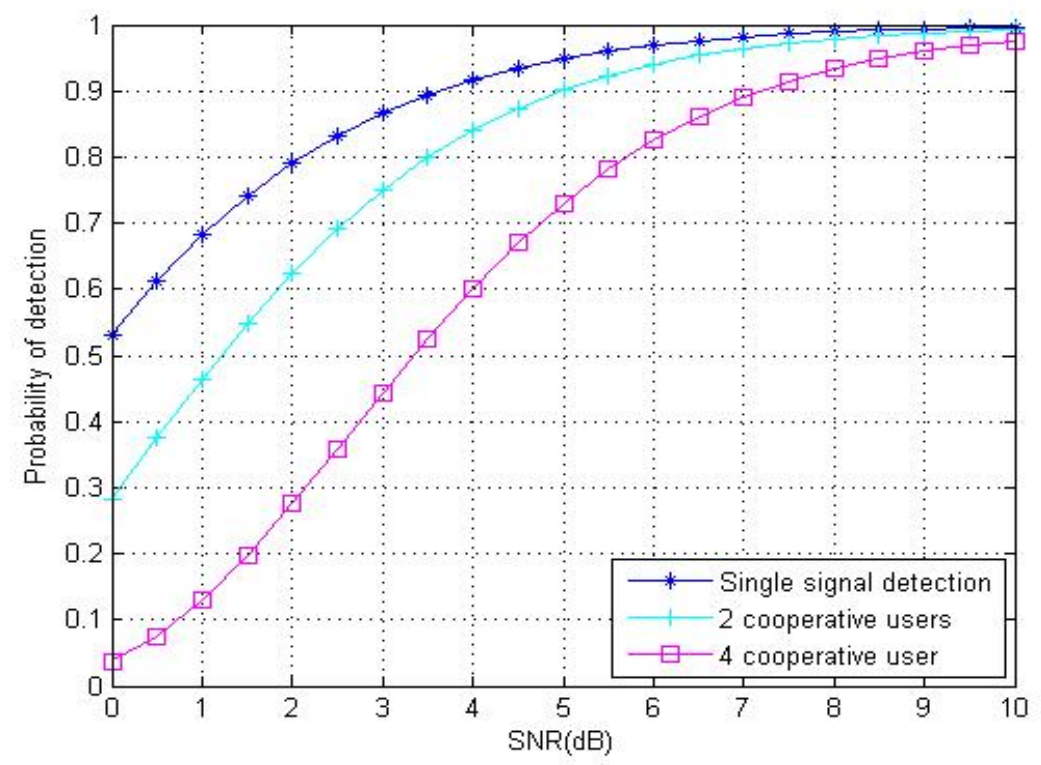

Figure 8 Probability of detection for AND rule.

Furthermore, improvement of detection probability by increasing number of collaborated user is shown in Figure 9. The simulation is conducted by varying number of cooperative users to confirm the previous results. We adopt OR rule and vary SNR values $0 \mathrm{~dB}, 1 \mathrm{~dB}$, and $2 \mathrm{~dB}$, respectively.

The result shows that increasing number of collaborated users that are populated in the range of primary transmitter can improve probability of detection in CR system. The probability of detection achieves an optimum value when number of collaborated users is greater than 15 . This result confirms that primary user 
signal can be detected more accurately by increasing number of collaborated users in cognitive radio.

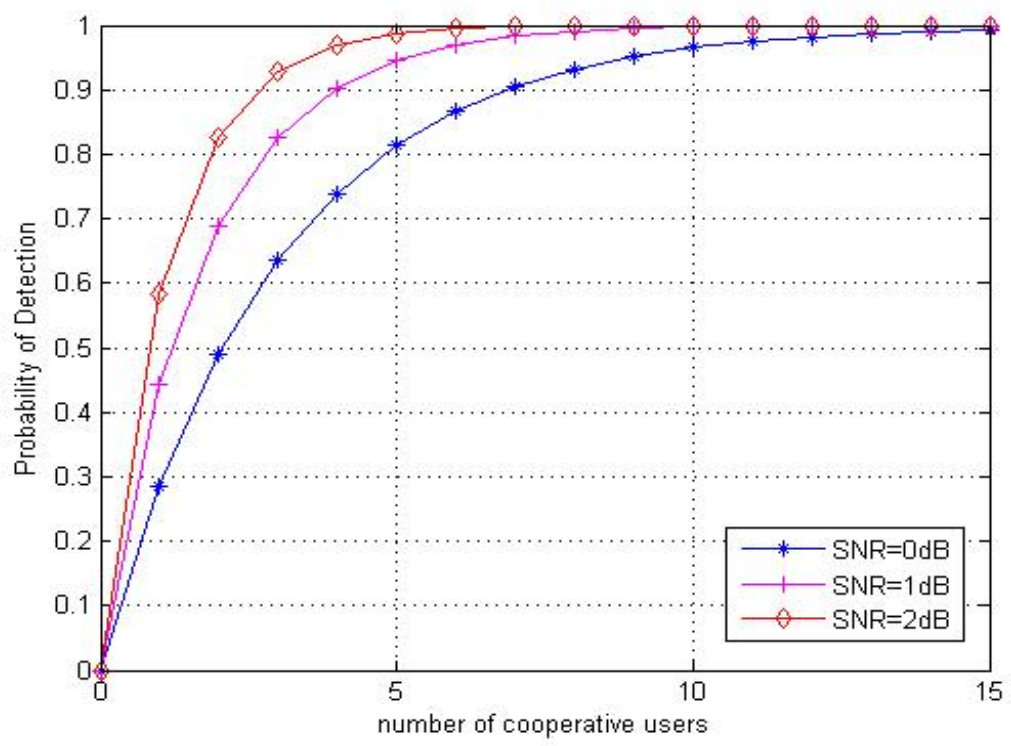

Figure 9 Number of collaborated users affects probability of detection in cognitive radio system.

\section{Conclusion}

We studied the performance of cooperative spectrum sensing and signal detection base on hard decision combining technique in data fusion centre compared with non-cooperative one. In cooperative technique, OR and AND rules are employed and evaluate the system performance by using probability of detection $\left(P_{d}\right)$ and SNR as metric. The OR rule decides $\mathrm{H}_{1}$ when at least one $\mathrm{CR}$ user forward bit- 1 while the AND rule decides $\mathrm{H}_{1}$ when all $\mathrm{CR}$ users forward their bit-1 to data fusion centre.

The numerical results show that cooperative technique has better performance compared with non cooperative one and employing OR rule can improve probability of detection than AND rule and non cooperative signal detection at different SNR values. Cooperative technique is more effective when received SNR in cognitive radio users is low due to fading and shadowing. Noncooperative technique achieves the same detection probability value (optimal value) as cooperative technique when received SNR is greater than $10 \mathrm{~dB}$, 
Furthermore, a minimum of 15 collaborated users relatively in cognitive radio system can achieve optimal value of detection probability. However, it depends on the threshold value used in signal detection.

\section{References}

[1] Yucek, T. \& Arslan, H., A Survey of Spectrum Sensing Algorithm for Cognitive Radio Applications, IEEE Communications Surveys \& Tutorials, 11(1), First Quarter 2009.

[2] Zheng, Y., Xie, X \& Yang, L., Cooperative Spectrum Sensing based on SNR comparison in Fusion Centre for Cognitive Radio, Proceedings of the 2009 International Conference on Advanced Computer Control, 2009.

[3] Varshney, P.K., Distributed Detection and Data Fusion, New York, Springer-Verlag, 1997.

[4] Visotsky, E., Kuffner, S. \& Peterson, R., On collaborative detection of TV transmissions in support of dynamic spectrum sharing, in Proc.IEEE Int. Symposium on New Frontiers in Dynamic Spectrum Access Networks, Baltimore, Maryland, USA, pp. 338-345, Nov. 2005.

[5] Weiss, T., Hillenbrand, J. \& Jondral, F., A diversity approach for the detection of idle spectral resources in spectrum pooling systems, in Proc. of the 48th Int. Scientific Colloquium, Ilmenau, Germany, pp. 37-38, Sept. 2003.

[6] Mishra, S., Sahai, A. \& Brodersen, R., Cooperative sensing among cognitive radios, in Proc. IEEE Int. Conf. Commun., 2, Istanbul, Turkey, pp. 1658-1663, May 2006.

[7] Sahai, A., Rahul, T., Mishra, S.M. \& Hoven, N., Fundamental Design Tradeoffs in Cognitive Radio Systems, ACM International Conference Proceeding Series, 222.

[8] Urkowitz, H., Energy Detection of Unknown Deterministic Signals, Proceedings of The IEEE, 55(4), April 1967.

[9] Ganesan, G. \& Li, Y., Agility improvement through cooperative diversity in cognitive radio, in Proc. IEEE Global Telecomm. Conf. (Globecom), 5, St. Louis, Missouri, USA, pp.2505-2509, Nov./Dec. 2005.

[10] Cabric, D., Tkachenko, A. \& Brodersen, R., Spectrum sensing measurements of pilot, energy, and collaborative detection, in Proc.IEEE Military Commun. Conf., Washington, D.C., USA, pp. 1-7, Oct. 2006.

[11] Leu, A., McHenry, M. \& Mark, B., Modeling and analysis of interference in listen-before-talk spectrum access schemes, Int. Journal of Network Management, 16, pp. 131-147, 2006.

[12] Ghasemi, A. \& Sousa, E.S., Collaborative spectrum sensing for opportunistic access in fading environments, First IEEE international Symposium on New Frontiers in Dynamic Spectrum Access Networks (DySPAN 2005), pp.131-136, Nov.2005. 
[13] Fadel, F.D., On the Energy Detection of Unknown Signals over Fading Channels, IEEE Transactions on Communications, 55(1), January 2007.

[14] D.Cabric, S.M.Mishar, and R.W.Brodersen, "Implementation Issues in Spectrum Sensing for Cognitive Radios," in Proc.of the IEEE ACSSC’04, Vol.1, Pacific Grove, CA, U.S.A, Nov.2004, pp.772-776. 